\title{
Outcome of DCS (Dynamic Condylar Screw) in failed proximal femur nails in intertrochanteric fractures: A retrospective study
}

\author{
Keyur Shah ${ }^{1}$, Abhishek Gol ${ }^{2, *}$, Dhimant Patel ${ }^{3}$, Hriday Acharya ${ }^{4}$, Ranjan Patariya ${ }^{5}$ \\ ${ }^{\mathbf{1}}$ Assistant Professor, ${ }^{\mathbf{2}, 4,5} 3^{\text {rd }}$ Year Resident, ${ }^{\mathbf{3}}{ }^{\text {nd }}$ Year Resident, Dept. of Orthopaedics, B.J. Medical College, Ahmedabad, \\ Gujrata, India
}

*Corresponding Author: Abhishek Gol

Email: abhishekkgol@gmail.com

\begin{abstract}
Introduction: Intertrochanteric Femur fractures comprise approximately more than half of hip fractures caused by low energy mechanism seen more commonly in geriatric people, women,osteoporosis, a history of fall and gait abnormality. There are surgical managments available for this kind of fractures but still there are some failures. Above mentioned factors are responsible for failures. For that revision surgery is required. Here we are going to search outcomes of DCS in failed PFN in IT fractures.

Materials and Methods: A retrospective study of 10 patients, treated with dynamic condylar screw (DCS) for implant failure after doing PFN in Intertrochanteric fracture were stastastically reviewed from march 2017 to march 2018.

Observation and Results: Out of 10 cases 4 were young ( $<60$ years) and 6 were elders ( $>60$ years). 4 out of 4 young patients and 3 out of 6 elder patients were united properly. Nonunion was seen in 1 patient. Delayed union was seen in 1 patient and Implant failure was seen in 1 cases $(10 \%)$. Restricted weight bearing status post-operatively was associated with significantly lower incidence of implant failure.Using the DCS, the results were good in the young patients with high-energy injuries. However, the implant failure rate is high in elderly patients who suffer low-energy fractures. The DCS should not be used if weight bearing cannot be minimized in this group.

Conclusion: The dynamic condylar screw is a safe and reliable implant for the management of Periimplant Subtrochanteric fractures in previously operated with PFN with predictable results when principles of open reduction and internal fixation is followed as compare to other methods of fixation in developing country.
\end{abstract}

Keywords: Proximal femur nail, Dynamic condylar screw, Intertrochentric fracture, Revision surgery.

\section{Intoduction}

Intertrochanteric

fractures

are extracapsular fractures of the proximal femur between the greater and lesser trochanters. This kind of fractures typically affects older patients than femoral neck fractures.

Intertrochanteric Femur fractures are most common hip fracture caused by low energy mechanism. These hip fractures occur in characteristic population with risk factors including increasing age, female gender, osteoporosis,a history of fall and gait abnormality.
Factors that associated with increase mortality are male gender, operative delay > 2days, age $>80$ yrs, preexisting medical conditons. Surgery within 2 days decrease mortality. In spite of latest techniques of fixation of trauma in last 50 years management of this fracture has always remained subject of debate. There are several internal fixation options for managing these fractures that generally fall into two categories: some form of intramedullary fixation or some form extramedullary fixation.
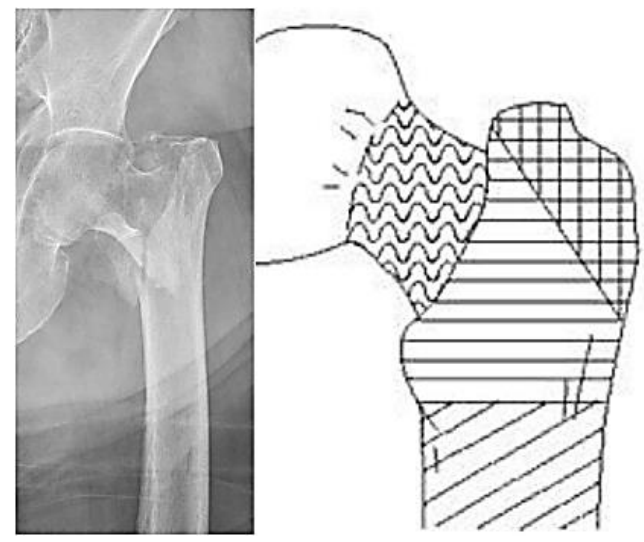

Subtrochanteric
region
II Greater
trodanter
Intertuochanteric
region
Femoral neck

AO classification of IT femur fracture 


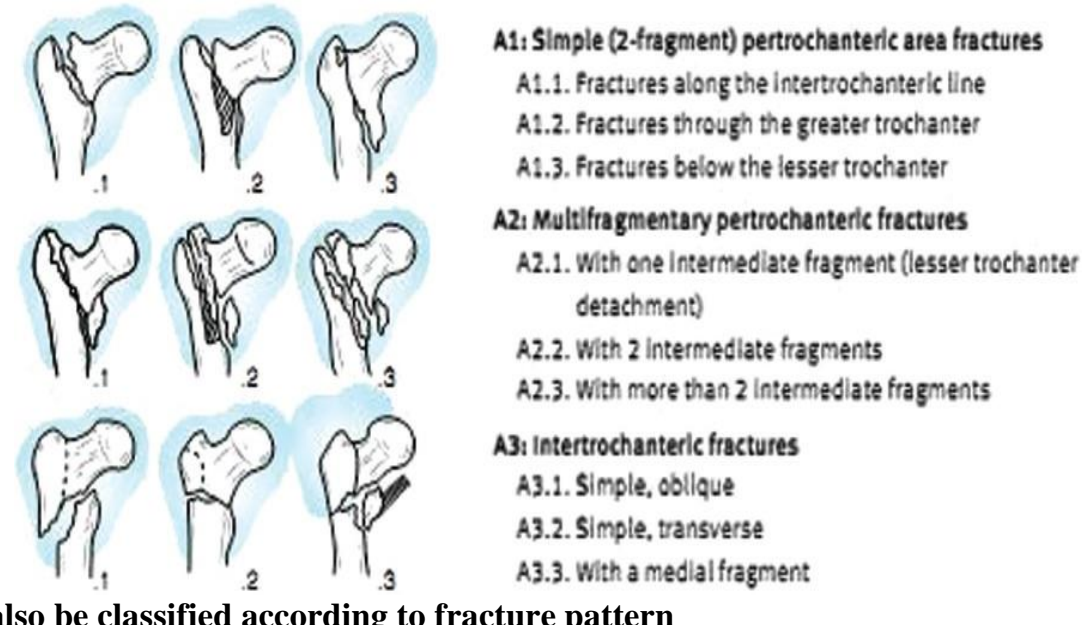

IT fracrtues can also be classified according to fracture pattern

IT fractures are classified as stable if posteromedial cortex is intact. Clinical significance of it is that It will resist medial compressive loads once reduced. IT fractures are classified as unstable if comminution of the posteromedial cortex is present. The stability of the fracture is inversely proportional to the size of the lesser trochanteric fragment. Instability occurs when more than $50 \%$ of the calcar is affected. Therefore, a fracture is considered unstable if there is a large lesser trochanteric fragment or if the greater and the lesser trochanter are separate fracture fragments (four-part fracture).Its clinical significance is that this type of fracture will collapse into varus and retroversion when loaded. Examples of unstable IT fractures are:

1. Fractures with a large posteromedial fragment. i.e., lesser trochanter is displaced

2. Subtrochanteric extension

3. Reverse obliquity. i.e., oblique fracture line extending from medial cortex both laterally and distally

\section{Operative Treatment for IT Fractures Includes}

1. sliding hip screw 2. intramedullary hip screw (cephalomedullary nail) 3. Arthroplasty 4. Dynamic condylar screw. Indications for sliding hip screw is stable intertrochanteric fractures with intact medial wall $^{11}$ while intramedullary hip screw (cephalomedullary nail) is used in stable fracture patterns, unstable fracture patterns, reverse obliquity fractures (where 55\% failure rate is found when treated with sliding hip screw), subtrochanteric extension and lack of integrity of femoral wall. This type of fracures are associated with increased displacement and collapse when treated with sliding hip screw.

Indications for arthroplasty are severely comminuted fractures, preexisting symptomatic degenerative arthritis,osteoporotic bone that is unlikely to hold internal fixation, salvage for failed internal fixation.

In Sliding hip compression screw type of fixation we must obtain correct neck-shaft relationship first. The research shows that the failure rates are increased with the lag screw with tip-apex distance $>25 \mathrm{~mm} .{ }^{11} 4$ hole plates show no benefit clinically or biomechanically over 2 hole plates. The only advantage of using 2 hole plate over 4 hole plate is shorter surgical time. ${ }^{12}$ Advantages of this type of fixation is that it allows dynamic interfragmentary compression. It has low cost. so can be used in low economic countries. Disadvanteges are that this is an open technique. That is why chances of blood loss and post operative infection are increased compare to intramedullary nailing. It is also not advisable in unstable type of IT fractures. Post operative complications includes limb shortening, collapse of fracture, leg screw cut-out and medialization of shaft. ${ }^{13}$

Intramedullary hip screw are of two types i.e. short implants and long implants. Indications of short implants are pertrochanteric fractures, intertrochanteric fractures and high subtrochanteric fractures. Contraindications are low subtrochanteric fractures, femoral shaft fractures, isolated or combined medial femoral neck fractures. Indications of long implants are low and extended subtrochanteric fractures, ipsilateral trochanteric fractures, combination of fractures (trochanteric area/shaft) and pathological fractures. Contraindications are isolated or combined medial femoral neck fractures. Advantages of intramedullary hip screw are that its approach is percutaneous, so chances of blood loss and infections are very less. It can be used in unstable IT fractures. Disadvantages are higher cost than sliding hip screw and periprosthetic fracture. ${ }^{13}$

Arthroplasty is advisable in old aged patients. Advantage of this technique is early full weight bearing. Disadvantages are increased blood loss and chances of infection, limb shortening/lengthening and dislocation of joint.

Indications for Dynamic Condylar Screw are transverse subtrochanteric fractures, short and long oblique subtrochanteric fractures. 
Choosing a most appropriate method for treating implant failure and nonunion in IT fracture is still under debate. ${ }^{1,3} 10-20 \%$ nonunion occurs following fixation of intertrochanteric fractures. ${ }^{5}$ Complications occur mainly in patients with osteoporosis, complicated fractures and improper fixation. ${ }^{1,5}$ Removal of implant followed by DCS with or without bone grafting can be used for implant failure while refreshing of fracture margins, debridement of fibrous tissue, correction of varus malalignment, autografting and fracture compression are essential for treating nonunion.

\section{Aims and Objectives}

To study results of DCS (dynamic condylar screw) in failed proximal nails in intertrochanteric fractures and evaluate treatment related complications.

\section{Materials and Methods}

The retrospective study was conducted at Civil Hospital, Ahmedabad, in patients of implant failure after doing PFN in Intertrochanteric fracture. We took 10 patients which were treated with Dynamic Condylar Screw (DCS) for implant failure after doing proximal femur nail. All these patients reviewed retrospectively. The mechanism of injury was high-energy in 7 cases and low-energy in 3 cases. 4 Out of 10 cases were young adults aged below 60 years and 6 were elders aged above 60years. 4 out of 4 young patients and 3 out of 6 elder patients were united properly. While other 3 elder patients are not united properly. One of them was gone into nonunion. One was presented with implant failure and delayed union was seen in the other one.

\section{Inclusion Criteria}

1. H/O PFN surgery in CHA

2. Patients who were in the age group of more than 25 years of either sex

3. Patients who are presented with either implant brackage or implant failure.

\section{Exclusion Criteria}

1. Patients unfit for the surgery

2. Open/ pathological/ bilateral fractures

3. Patients who had less than 2 years of follow-up

4. Polytrauma patients

5. Pre-existing hip/femoral deformity

6. Patients with infection

\section{Technique Used}

Dynamic Condylar Screw: On fracture table,in supine position, under anaesthesia, painting and draping had done. An incision made over previously present stichline scar. After removal of proximal and distal screws, a PFN was removed. Reduction of fracture was done under C-Arm. Incision then extended distally, cut TFL and vastus lateralis was lifted. Further reduction was made by using spikes, GT and shaft exposed. After introducing of condylar screw, a plate was placed and fixed by HCS and FT screw. Tension band wiring had done for further reduction of fracture. Closure done in layers. Drain kept. Dressing had put.

Post operative Care and Rehabilitation: Immediate post operative check x-ray should be done in both AP and cross table views to confirm reduction. Toes, ankle, knee and hip mobilization started on post operative day. Patient advised exercise on discharge. Suture removed after 15 days. Check x-ray was done after 1.5 month and see for union. Weight bearing allowed after it. Follow up is done at 1.5 month, 3 month and 6 month. Range of motion and deformity checked.

\section{Observation and Results}

In this study we have taken 10 patients which were treated for IT fracture and PFN were done previously and presented with implant failure or implant brackage. Out of 10 cases 4 were young ( $<60$ years) and 6 were elders (>60years). 3 out of 10 cases were due to trival trauma (fall by slipping), all 3 were elders. While other 7 were due to high velocity trauma (RTA and Fall from height). Implant breakage occurred in all 4 young patients were due to high velocity trauma. All 10 patients were treated with implant removal and DCS.

In one year follow up, 7 out of 10 cases showed union. All 4 young patients showed union in their 1 year follow up xrays. Out of 10,1 was going into delayed union, 1 was malunited and 1 had been gone into non union. All these 3 were elder ( $>60$ years age) patients.

Fig. 1,2,3,4,5 shows serial xrays of 50 yrs old female patient with intertrochanteric fracture operated at civil hospital,Ahmedabad
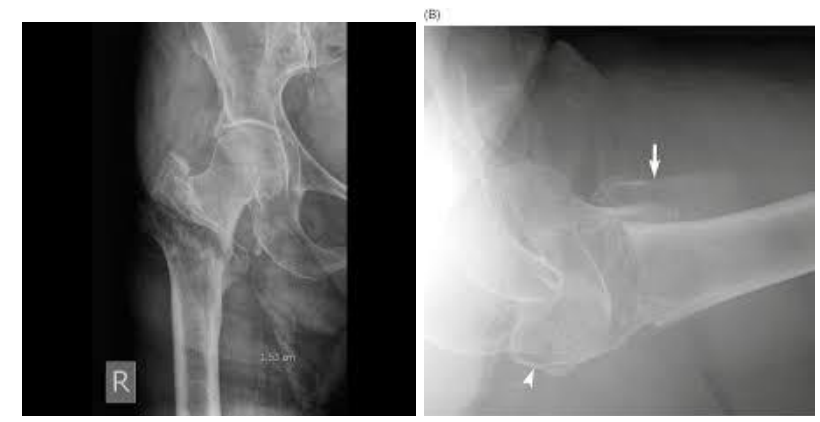

Fig. 1: AP and cross table radiograph of Hip with Femur Rt side demonstrating Intertrochanteric femoral fracture in 50 yr old female patient 


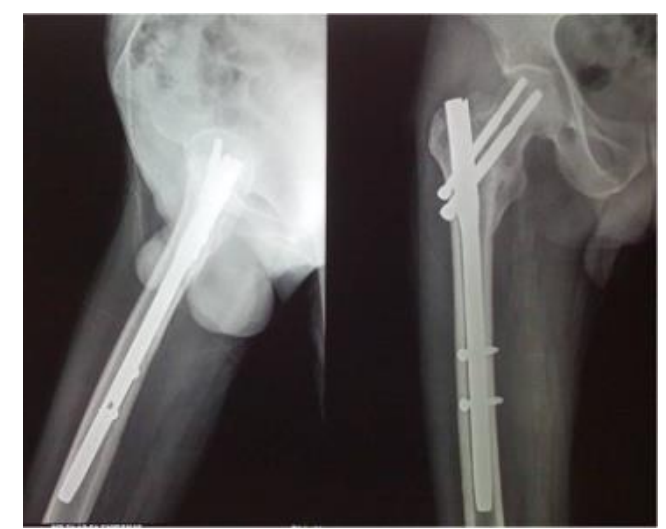

Fig. 2: AP and crosstable radiograph demonstrating fixation with a short Proximal Femur Nail after 2 days of trauma

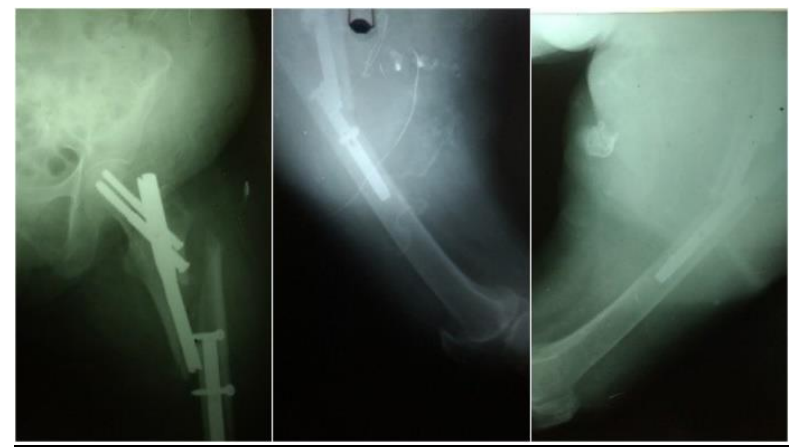

Fig. 3: Radiographs shows periimplant subtrochanteric femur fracture with implant brackage.(patient had history of trauma again after 3 months of primary fixation)

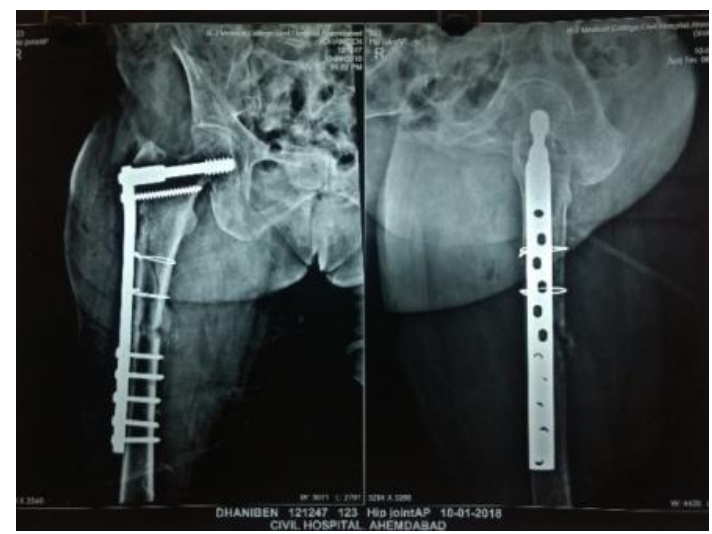

Fig. 4: AP and crosstable immidiate postop radiographs demonstrating reduction and stabilization. (Secondary fixation after removal of short proximal femur nail and fixation achived by dynamic condylar screw and tension band wiring.)

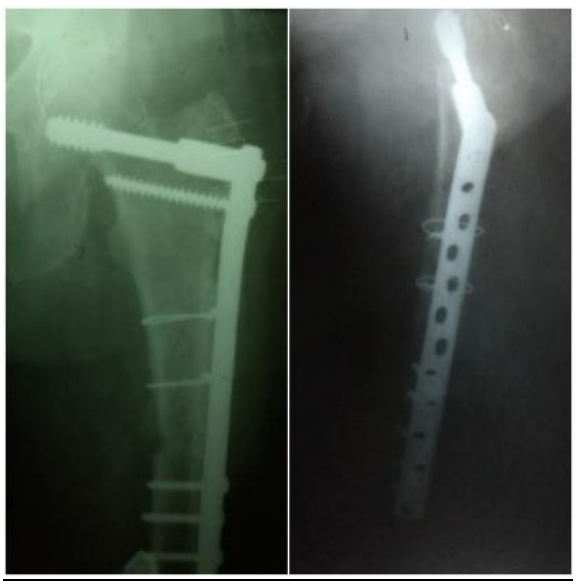

Fig. 5: Radiographs after 6 weeks shows calus formation and healing of fracture

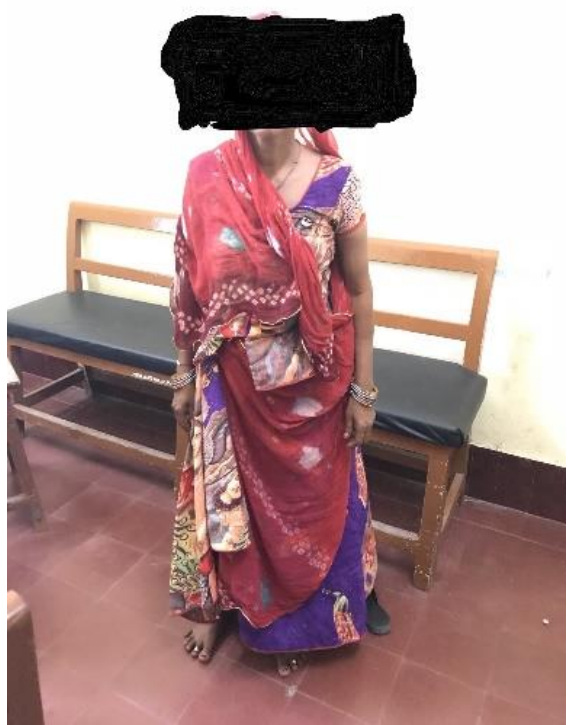

Fig. 6: Clinical image of patient bearing weight on both lower limbs after 2 months of fracture fixation

Here as required written consent of patients and relatives was taken.

Graph 1: Age of patients

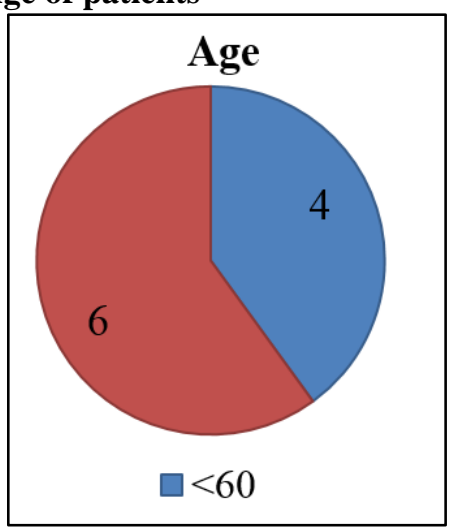




\section{Graph 2: Mode of trauma}

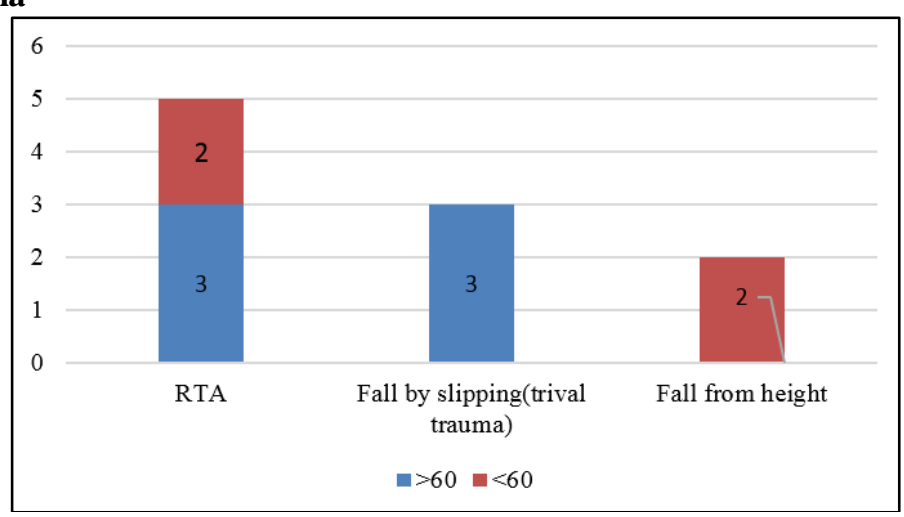

Graph 3: After 1 year of follow up

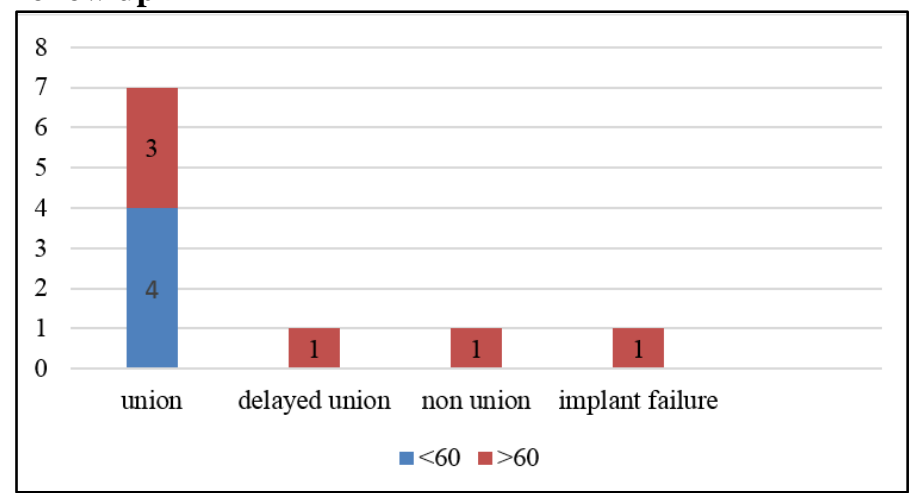

\section{Discussion}

Intertrochanteric fractures of femur occurs between a greater trochanter where hip extensors and hip abductors are attached and lesser trochanter where hip flexor is attached. Earlier it was thaught that IT fractures routinely healed but in many cases they were malunited in varus, leading to decreased function, deformity, pain and limp. Conservative treatment also leads to morbidity and mortality due to prolonged incumbency. Because of these problems the role of conservative management is gradually decreased. The other problem related to intertrochanteric fractures is the associated osteoporosis or osteopenia seen in the aged population. So the goal of the treatment of IT fractures are decrease the possibility of malunion and nonunion, decrease morbidity, early mobilization and achieve union in elder patients. That is why surgical approach is considered more suitable for IT fractures. IT fractures occurs as a results of low energy trauma (seen in elder patients;more common) and high energy trauma (seen in young patients; rare). The etiology of low energy IT fractures are increased fragility secondary to osteoporosis and osteomalacia. It is due to aging process, benign and malignant tumors along with metastases, decreased calcium and Vit D3 intake, decreased hormone level after menopause,prolonged bed ridden. Surgical treatment for IT fractures are sliding hip screws, cephalomedullary nailing, arthroplasty, dynamic condylar screw, which are very according to fracture patterns and age of patients. The complications of surgically treated IT fractures are nonunion, malunion, periimplant fracture with implant breakage, implant failure, screw back-out, infection. Treatment modalities of above mentioned complications are different. ${ }^{13}$ Periimplant Subtrochanteric fractures/Implant breakage/Nonunion in previously operated with PFN requires specific attention as there are high rate of complications rates. ${ }^{9}$ Intramedullary devices are unique in sense that they reduces bending forces. ${ }^{7}$ But they can't be used properly where medial cortex comminuations are present. Other methods like blade plate and kuntscher rods are better to use in this kind of fractures.Subtrochanteric fractures can't properly fixed with sliding hip screw because they don't provide additional proximal fragment fixation. ${ }^{10}$

Due to non availability of image intensifier in developing country dynamic condylar screw gives a better alternative. Also condylar screw gives additional advantage of fixing proximal fragment with extra screws. Biological fixation can be achived with condylar blade plate but technique is difficult. DCS provide better sagittal plane and rotation adjustments. DCS can't fix medial cortex properly but vascularity of small medial fragments are not altered which results in better union. Type $\mathrm{C} 1$ and $\mathrm{C} 2$ fractures are difficult to reduce. Types $\mathrm{A}$ and $\mathrm{B}$ fractures can be reduced with traction and internal rotation only. 
11 though our study included relatively small number of cases, we avoid selection bias in almost all cases.

\section{Conclusion}

DCS is better and safe implant for perimplant subtrochanteric fractures in previously treated with PFN compare to other methods of fixation in developing countries due to cost effectiveness and non availability of image intensifier.

\section{Abbreviations}

DCS: Dynamic Compression Screw; IMD: intramedullary device; TGN: trochanteric gamma nail.

\section{References}

1. Lavelle David G (2003) Campbells operative orthopaedics, 10th edn. Mosby, St. Louis, p 2897.

2. Asher MA, Tippet JW, Rockwood CA, Zilber S (1976) Compression fixation of subtrochanteric fractures. CORR No 117: June.

3. Sims SH. Treatment of complex fractures. Orthop Clin North Am. 2002;33(1):1-12.

4. Kinast C, Bolhofner BR, Mast JW, Ganz R. Subtrochanteric fractures of the femur. Results of treatment with the 950 condylar blade plate. Clin Orthop. 1989;238:122.

5. Muller ME, Nazarian S, Koch P et al (1990) The AO classification of fractures of long bones. Springer, Berlin, p 116

6. Tencer AF, Johnson KD, Johnston DW. A biomechanical comparison of various methods of stabilization of subtrochanteric fractures of femur. J Orthop Res. 1984;2:297-305.

7. Fielding JW, Magliato HJ (1966) Subtrochanteric fractures. Surg Gynaecol Obstet. 1966;122:297-305.

8. Bedi A, Le TT. Subtrochanteric femur fractures. Orthop Clin North Am. 2004;35:473-483.
9. Warwick DJ, Crichlow TPKR, Langkamer VG. The dynamic condylar screw in management of subtrochanteric fractures of the femur. Injury. 1995;26(4):241-244.

10. Imran Haruna Abdulkareem, A review of tip apex distance in dynamic hip screw fixation of osteoporotic hip fractures, Niger Med J. 2012;53(4):184-191. doi: 10.4103/0300-1652.107550

11. Říha, D. \& Bartoníček, Internal fixation of pertrochanteric fractures using DHS with a two-hole side-plate, J. International Orthopaedics (SICOT). 2010;34:877. https://doi.org/10.1007/s00264-009$0840-\mathrm{Z}$

12. Zorik Avakian, Timothy Shiraev, Lawrence Lam and Nigel Hope, Dynamic hip screws versus proximal femoral nails for Intertrochanteric Fractures, ANZ Journal of Surgery @ 2011 Royal Australasian College of Surgeons, doi: 10.1111/j.1445-2197.2011.05929.x

13. Robinson Esteves Santos Pires, Egídio Oliveira Santana Júnior, Leandro Emilio Nascimentro Santos Vincenzo Giordano, Daniel Balbachevsky and Fernando Baldy dos Reis, Failure of fixation of trochanteric femur fractures: Clinical recommendations for avoiding Z-effect and reverse Z-effect type complications, Patient Safety in Surgery. 20115:17 https://doi.org/10.1186/1754-9493-517

14. (C) Pires et al; licensee BioMed Central Ltd. 2011.

How to cite this article: Shah $\mathrm{K}$, Gol A, Patel D, Acharya H, Patariya R. Outcome of DCS (Dynamic Condylar Screw) in failed proximal femur nails in intertrochanteric fractures: A retrospective study. Indian J Orthop Surg. 2018;4(4):310-315. 